\title{
Is Normal Coronary Arteries on Coronary Angiogram Truly Benign?
}

\author{
Indrani Garre ${ }^{1}$ Raju Nallagasu² $\quad$ Lalita Nemani $^{1} \quad$ Sreebhushanraju Devaraju ${ }^{2} \quad$ Narendrakumar R. N. V. ${ }^{1}$ \\ Neeraja Kakamanu ${ }^{1}$
}

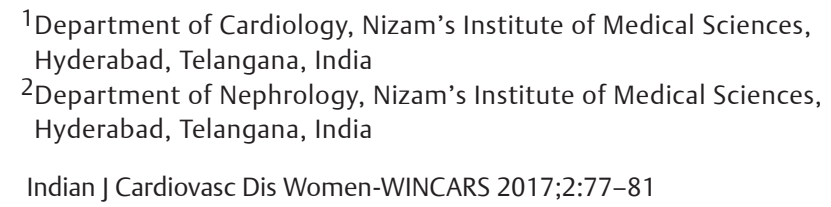

Address for correspondence Indrani Garre, MSc, PhD Student, Department of Cardiology, Nizam's Institute of Medical Sciences, Hyderabad, TS 500082, India (e-mail: indrani.garre@gmail.com).

\section{Abstract}

Keywords

- blood urea

- normal coronaries

- coronary angiogram
Background Heart disease is the leading cause of death in the world, and coronary angiography (CAG) is the standard test for detection of critical diseases. Nearly $25 \%$ patients undergoing angiography are found to have normal coronary arteries (NCAs). Recent publications say that the follow-up of the NCA patients is not benign.

Aim The main objective of this study is to know outcomes of patients diagnosed with normal coronaries on conventional angiograms.

Methods This is a single-center observational study. The authors collected the data of patients who came for CAG to their institute from January 1 to December 31, 2014. They included the patients who have normal coronaries in this study. The indication to do the CAG is chest pain (angina) for evaluation. The authors contacted every patient telephonically to know the outcomes after 2 years of CAG. The telephonic data were collected either from patients or from attendants by asking the questionnaire about the present status of patients.

Results The authors collected the data of 1,526 patients who underwent the CAG during study period. Out of 1,526 patients, 201 patients with mean age of $52.45 \pm 10.7$ years met the inclusion criteria; 108 (53.73\%) were hypertensives and 57 (28.36\%) were diabetic. Acute coronary syndrome (ACS) was the presenting symptom in $28(13.9 \%)$ of patients; the remaining patients presented with chronic stable angina (CSA). Left ventricular (LV) dysfunction was present in 15 (7.5\%) of patients. At the 2-year follow-up, 12 (5.9\%) patients were symptomatic, and out of them, 7 died (mortality 3.5\%).

The binary logistic regression showed that only blood urea (BU) was determinant factor for mortality $(p=0.022)$. Even LV dysfunction $(p=0.39)$ was not a contributor for mortality. A subanalysis of gender also showed similar findings (male, $p=0.02$; female, $p=0.05)$.

Conclusion In angina patients with NCA on CAG, the BU showed statistical significance for morality. This is true even on further sex-based analysis (male, $p=0.02$; female, $p=0.05$ ). This study helps understand that even the people with normal coronaries, they may have the symptomatic outcomes, not only because of coronaries and also with other associated diseases.
DOI https://doi.org/ 10.1055/s-0038-1622962.
Copyright @2017 Women in Cardiology and Related Sciences
License terms

()ㅜ(1) $\Theta \circledast$ 


\section{Introduction}

Heart disease is the leading cause of death in the world, and coronary angiography (CAG) is the standard test for detection of critical diseases. ${ }^{1}$ Nearly $25 \%$ patients undergoing angiography are found to have normal coronary arteries (NCAs). Recent publications say that the follow-up of the NCA patients is not benign. The NCA could lead to undertreatment of modifiable risk factors that may lead to coronary heart disease (CHD) latter. In patients admitted with ACS diagnosis, but with insignificant coronary artery disease or NCA, there is approximately a $2 \%$ risk of death or reinfarction over 6 to 12 months., ${ }^{2,3}$,

Interestingly, ${ }^{4-6}$ some patients with ST-elevation myocardial infarction (MI) have NCA anatomy at CAG. This situation is called MINCA (acute myocardial infarction with normal coronary artery). The incidence of MINCA varies between 1 and $8.5 \%$. In fact, 2.6 to $12 \%$ of patients who undergo CAG because of MI with suspected ischemic heart disease are found to have nearly NCAs in angiography. ${ }^{7,8}$ The prognosis of NCAs with suspected signs and symptoms of ischemia is not really benign as reported by previous studies and as commonly assumed by physicians. ${ }^{9}$ Although NCAs were recognized angiographically more than 30 years ago, ${ }^{10}$ the malignant nature of the outcomes is being discussed recently only.

\section{Materials and Methods}

This is a single-center observational study. The authors collected the data of patients who came for CAG to their institute from January 1 to December 31, 2014. They included the patients who have normal coronaries in this study. The indication to do the CAG is chest pain (angina) for evaluation. The authors excluded the patients in whom the CAG was done for other reasons, such as planned surgery in vulvar diseases, fitness for renal transplants, and different cardiomyopathies (as the outcomes depended on type of cardiomyopathies) such as dilated cardiomyopathies and hypertrophic obstructive cardiomyopathy. The authors noted the demographic features such as age, sex, CAD risk factors, type of CAD (acute coronary syndrome [ACS] or chronic stable angina [CSA]), electrocardiogram (ECG), two-dimensional (2D) echo parameters, and laboratory parameters such as complete hemogram, renal and hepatic function tests, and CAG findings. They followed the patients for 2 years. They contacted every patient telephonically to know the outcomes after 2 years of CAG. The telephonic data were collected either from patients or from attendants by asking the questionnaire about the present status of patients. If the patient developed angina, dyspnea, or syncope, or required hospitalization for cardiac cause, or any coronary revascularization (morbidity), or death (mortality) in these 2 years of follow-up, they were considered as symptomatic. Telephonically the authors could get the information about the death of the patients by relatives but were not able to get proper information about the cause of the death.

\section{Statistical Analysis}

The results were expressed as mean \pm SD (standard deviation) for continuous data and number and percentages for categorical data. The assumption of normality was sustained in all the data. Binary logistic regression was done to see the significant factors contributing to the mortality. One-way ANNOVA (analysis of variance) was done for repeated measures, and contingency tables were used to compare findings of results. A p value of $<0.05$ was considered significant.

The statistical analysis was done by Minitab software version 17 by Minitab, Inc., Coventry, United Kingdom.

\section{Results}

The authors collected the data of 1,526 patients who underwent the CAG in their institute from January 1 to December 31 , 2014. Out of 1,526 patients, 318 (20.88\%) were diagnosed with normal coronaries. The authors were able to follow up telephonically with 220 (69.1\%).

Out of the 220 follow-up cases of NCA, inclusion criteria were fulfilled in 201 (91.36\%) patients and 19 (8.64\%) patients were excluded. As the main objective of this study is to know outcomes on patients diagnosed with coronary disease, this study population is 201. The descriptive statistics of patients with coronary diseases is described in - Table $\mathbf{1}$. Almost equal number of males and females were included. In 15 patients of LV dysfunction, patients had previous history and ECG evidence of old MI. Severity of LV dysfunction was mild in nine, moderate in two, and severe in four patients.

In - Table 2, some of the biochemical and cardiac catheterization parameters are mentioned. The systolic, diastolic, and pulse pressures were derived during cardiac catheterization.

Twelve patients were symptomatic, and out of them, mortality was in seven (3.5\%) and morbidity was in five (2.5\%), represented in - Table $\mathbf{3}$. Out of seven mortality cases, two had LV dysfunction (one patient had moderate and one had severe LV dysfunction).

The binary logistic regression was done to see which parameters determine the symptomatic status that includes both

Table 1 Descriptive statistics for patients with coronary disease

\begin{tabular}{|l|l|}
\hline Variables & Value \\
\hline Total No. of patients & 201 \\
\hline Male:Female & $102(50.75 \%): 99(49.25 \%)$ \\
\hline Age $(y)$ & $52.45 \pm 10.97$ \\
\hline Hypertension & $108(53.73 \%)$ \\
\hline Diabetes mellitus & $57(28.36 \%)$ \\
\hline Smoking & $22(10.9 \%)$ \\
\hline ACS & $28(13.9 \%)$ \\
\hline CSA & $173(86.1 \%)$ \\
\hline Family history & $12(5.9 \%)$ \\
\hline LV dysfunction & $15(7.5 \%)$ \\
\hline
\end{tabular}

Abbreviations: ACS, acute coronary syndrome; CSA, chronic stable angina; LV, left ventricular. 
Table 2 Biochemical and cardiac catheterization parameters of study population

\begin{tabular}{|l|l|}
\hline Variable & Mean \pm SD \\
\hline Systolic pressure $(\mathrm{mm} \mathrm{Hg})$ & $148.84 \pm 26.55$ \\
\hline Diastolic pressure $(\mathrm{mm} \mathrm{Hg})$ & $74.28 \pm 12.83$ \\
\hline Pulse pressure $(\mathrm{mm} \mathrm{Hg})$ & $73.9 \pm 24.29$ \\
\hline Serum creatinine $(\mathrm{mg} / \mathrm{dL})$ & $0.97 \pm 0.61$ \\
\hline Blood urea $(\mathrm{mg} / \mathrm{dL})$ & $24.39 \pm 12.32$ \\
\hline
\end{tabular}

Abbreviation: SD, standard deviation.

Table 3 Details of outcomes

\begin{tabular}{|l|l|}
\hline Outcome & No. (\%) \\
\hline Symptomatic & $12(5.9 \%)$ \\
\hline Mortality & $7(3.5 \%)$ \\
Morbidity & $5(2.5 \%)$ \\
\hline
\end{tabular}

Table 4 Binary logistic regression for mortality

\begin{tabular}{|l|l|l|}
\hline Parameters & Chi-square & $\boldsymbol{p}$ Value \\
\hline Age $(\mathrm{y})$ & 2.41 & 0.12 \\
\hline Blood urea $(\mathrm{mg} / \mathrm{dL})$ & 5.22 & 0.02 \\
\hline Serum creatinine $(\mathrm{mg} / \mathrm{dL})$ & 3.53 & 0.06 \\
\hline Systolic pressure $(\mathrm{mm} \mathrm{Hg})$ & 0.02 & 0.88 \\
\hline Diastolic pressure $(\mathrm{mm} \mathrm{Hg})$ & 0.00 & 0.95 \\
\hline Radial pulse pressure $(\mathrm{mm} \mathrm{Hg})$ & 0.19 & 0.66 \\
\hline Sex & 0.36 & 0.55 \\
\hline Hypertension & 0.25 & 0.62 \\
\hline Diabetics mellitus & 0.81 & 0.37 \\
\hline Smoker & 0.10 & 0.75 \\
\hline LV dysfunction & 0.74 & 0.39 \\
\hline
\end{tabular}

Abbreviation: LV, left ventricular.

morbidity and mortality and separately only for mortality. In this analysis, the authors found that blood urea (BU) had a significant association with symptomatic status $(p=0.049)$ and mortality $(p=0.022)$. - Table 4 represents the results of binary logistic regression for mortality. Surprisingly, even LV dysfunction was not a contributing factor for mortality ( $p=0.39$ ).

For mortality in this regression analysis, sex was not a contributing factor. The authors want to see whether the same factors contribute in males and females separately. One-way ANOVA showed BU as determinant factor for mortality in both (male, $p=0.02$, female, $p=0.05$ ) sexes.

\section{Discussion}

In the late 1980s itself, normal coronaries on CAG were known to occur in ACSs and in CSAs. ${ }^{10}$ Since then, various series of such patients have been described, but the etiology and pathogenesis of the condition are still a source of debate. Evidence exists for a role of coronary vasospasm, thrombosis, embolization, and inflammation, per se or combined may be responsible. ${ }^{11}$
In general, NCA was considered as benign course. However, recent data from studies by McMullan et $\mathrm{al}^{1}$ and Bugiardini et $\mathrm{al}^{2}$ have shown that event rates are $10.1 \%$ and $2 \%$ event in NCA group. According to those authors, the determinants for events were cardiac, multiorgan system failure, ischemic stroke, and malignancy. However, in this series, the authors did not find these factors significant either in multi- or univariate analysis. Instead, the authors found in both uni- and multivariate analyses that there was strong correlation with renal impairment with symptomatic status.

Renal dysfunction has been associated with adverse cardiovascular outcomes. ${ }^{12-15}$ Notably, the association between BU and cardiovascular outcomes remained significant across strata of other biomarkers associated with increased cardiovascular risk, namely troponin-I, brain natriuretic peptide (BNP), and C-reactive protein (CRP). The main findings from the study by Kirtane et al revealed that BU was a significant predictor of long-term mortality in patients who underwent percutaneous coronary intervention $(\mathrm{PCI})$ regardless of the presence of ACS or stable CAD. Especially, a BU of $25 \mathrm{mg} / \mathrm{dL}$ was associated with long-term mortality independent of estimated glomerular filtration rate (eGFR) and other clinical characteristics. In addition, the association between BU levels and mortality was evident at values of 20 to $25 \mathrm{mg} / \mathrm{dL}$, suggesting that even minimal elevations in BU may be a marker of adverse outcomes. ${ }^{16} \mathrm{BU}$ has been associated with adverse outcomes and has been previously incorporated into MI risk prediction models. ${ }^{17,18}$ Gotsman et al ${ }^{19}$ suggested that serum urea is a more powerful predictor of survival than eGFR in patients with heart failure (HF).

Even though renal dysfunction is reflected better by GFR value than BU (mentioned in the limitations of the study), $\mathrm{BU}$ also has its role in predicting the renal function. BU levels may provide supplemental information regarding renal function as renal proximal tubule cells may increase BU reabsorption in the setting of increased neurohormonal activation. The study by Ostfeld et al suggests that an elevated serum BU in patients presenting with signs and symptoms of unstable angina and without known CAD may predict a larger burden of CAD on cardiac catheterization independent of creatinine clearance. $^{20}$

This prospective study describes the outcome at 2-year follow up for 201 angiographically NCA patients. In this study, authors analyzed the clinical parameters along with angiogram findings which may influence the outcomes. They suggested that even though patients were diagnosed with no obstructive coronary artery disease angiogram wise, it is better to take clinical parameters also to know the prognosis of these patients as this study demonstrated increasing cases of death and morbidity in angiogramdiagnosed NCA patients.

Another important factor in NCA is really that there were normal coronaries without any atherosclerosis. The accuracy of angiography has been questioned for a long time. Investigations with intravascular ultrasound show high-risk lesions in many vessels with no apparent disease seen via standard angiography; these lesions are at risk of 
rupture and acute thrombus formation despite negligible luminal stenosis. False-negative angiograms could lead to undertreatment of modifiable risk factors or failure to protect patients with CHD from life-threatening cardiac events. ${ }^{11}$

\section{Limitation of the Study}

The first major limitation of this study is that BU, but not GFR was used as a marker for mortality in NCA patients. The blood urea nitrogen (BUN) concentration has been considered a less specific marker of kidney function than serum creatinine $(\mathrm{sCr})$, estimated creatine clearance, or eGFR. However, in addition to reflecting GFR, BU may rise independent of changes in GFR or sCr owing to enhanced proximal tubular reabsorption under activation of the sympathetic nervous and renin-angiotensin-aldosterone systems. ${ }^{21}$

Second, it is a single-center observational study with a small population. This analysis is a nonrandomized observational analysis, and as such, it is possible that both identified and unidentified confounders may have influenced the outcomes.

In conclusion, the BU role in cardiovascular disease mortality is shown in few studies, and as per this study, BU showed significant outcomes in the follow-up of patients who were diagnosed with normal coronaries. CAG accuracy in finding the diseased myocardium at smaller vessels level is still questionable. As per previous studies, an increase in BU levels will be associated with cardiovascular disease progression along with renal impairments.

In future, the authors need to concentrate on BU as it plays an important role in mortality that still needs to justify more with higher population. Even though, in addition to the evaluation of conventional risk factors in daily clinical practice, the measurement of $\mathrm{sCr}$ and $\mathrm{BU}$ level might provide significant prognostic benefits in terms of global cardiovascular risk and management of the patients.

\section{Conclusion}

According to this study, the BU showed statistical significance for mortality in NCA patients on CAG. The further sex-based analysis also showed that BU had statistical significance as predictor of mortality. This study helps understand that even people with normal coronaries may have the symptomatic outcomes, not only because of coronaries but also with other associated diseases.

\section{Acknowledgments}

The authors would like to thank Prof Maddury Jyotsna for guidance and encouragement.

\section{References}

1 McMullan JT, Lindsell CJ, Blomkalns AL. Five-year mortality and coronary heart disease development after normal coronary angiogram. World J Emerg Med 2011;2(1):24-29

2 Bugiardini R, Manfrini O, De Ferrari GM. Unanswered questions for management of acute coronary syndrome: risk stratification of patients with minimal disease or normal findings on coronary angiography. Arch Intern Med 2006;166(13):1391-1395

3 Patel MR, Chen AY, Peterson ED, et al. Prevalence, predictors, and outcomes of patients with non-ST-segment elevation myocardial infarction and insignificant coronary artery disease: results from the Can Rapid risk stratification of Unstable angina patients Suppress ADverse outcomes with Early implementation of the ACC/AHA Guidelines (CRUSADE) initiative. Am Heart J 2006;152(4):641-647

4 Celik M, Akil M, Tuncer M. Acute myocardial infarction with normal coronary artery (MINCA) in a patient with multiple sclerosis. World J Cardiovasc Dis 2013;3(8):483-486

5 Thygesen K, Alpert JS, Jaffe AS, et al; Joint ESC/ACCF/AHA/ WHF Task Force for Universal Definition of Myocardial Infarction; Authors/Task Force Members Chairpersons; Biomarker Subcommittee; ECG Subcommittee; Imaging Subcommittee; Classification Subcommittee; Intervention Subcommittee; Trials \& Registries Subcommittee; Trials \& Registries Subcommittee; Trials \& Registries Subcommittee; Trials \& Registries Subcommittee; ESC Committee for Practice Guidelines (CPG); Document Reviewers. Third universal definition of myocardial infarction. J Am Coll Cardiol 2012;60(16):1581-1598

6 Berger PB, Ellis SG, Holmes DR Jr, et al. Relationship between delay in performing direct coronary angioplasty and early clinical outcome in patients with acute myocardial infarction: results from the global use of strategies to open occluded arteries in Acute Coronary Syndromes (GUSTO-IIb) trial. Circulation 1999;100(1):14-20

7 Panza JA. Myocardial ischemia and the pains of the heart. N Engl J Med 2002;346(25):1934-1935

8 Magnoni M, Esposito A, Coli S, et al. IMAGE CARDIO MED: Two different mechanisms of myocardial ischemia involving 2 separate myocardial segments in a patient with normal coronary angiography. Circulation 2010;121(1):e1-e3

9 Mallinson T. Myocardial infarction. Focus on First Aid. 2010;15:15

10 Kardasz I, De Caterina R. Myocardial infarction with normal coronary arteries: a conundrum with multiple aetiologies and variable prognosis: an update. J Intern Med 2007; 261(4):330-348

11 Anavekar NS, McMurray JJ, Velazquez EJ, et al. Relation between renal dysfunction and cardiovascular outcomes after myocardial infarction. N Engl J Med 2004;351(13):1285-1295

12 Dossetor JB. Creatininemia versus uremia. The relative significance of blood urea nitrogen and serum creatinine concentrations in azotemia. Ann Intern Med 1966;65(6):1287-1299

13 McCullough PA. Interface between heart disease and renal dysfunction: From association to action. ACC Curr J Rev 2003;12:20-24

14 Aronson D, Mittleman MA, Burger AJ. Elevated blood urea nitrogen level as a predictor of mortality in patients admitted for decompensated heart failure. Am J Med 2004; 116(7):466-473

15 Akanda MAK, Choudhury KN, Ali MZ, et al. Serum creatinine and blood urea nitrogen levels in patients with coronary artery disease. Cardiovascular J 2013;5(2):xx

16 Kirtane AJ, Leder DM, Waikar SS, et al; TIMI Study Group. Serum blood urea nitrogen as an independent marker of subsequent mortality among patients with acute coronary syndromes and normal to mildly reduced glomerular filtration rates. J Am Coll Cardiol 2005;45(11):1781-1786

17 Luria MH, Knoke JD, Margolis RM, Hendricks FH, Kuplic JB. Acute myocardial infarction: prognosis after recovery. Ann Intern Med 1976;85(5):561-565

18 Normand ST, Glickman ME, Sharma RG, McNeil BJ. Using admission characteristics to predict short-term 
mortality from myocardial infarction in elderly patients. Results from the Cooperative Cardiovascular Project. JAMA 1996; 275(17):1322-1328

19 Gotsman I, Zwas D, Planer D, Admon D, Lotan C, Keren A. The significance of serum urea and renal function in patients with heart failure. Medicine (Baltimore) 2010;89(4):197-203
20 Ostfeld R, Spinelli M, Mookherjee D, et al. The association of blood urea nitrogen levels and coronary artery disease. Einstein J Biol Med 2016;25:3

21 Usberti M, Federico S, Di Minno G, et al. Effects of angiotensin II on plasma $\mathrm{ADH}$, prostaglandin synthesis, and water excretion in normal humans. Am J Physiol 1985;248(2 Pt 2):F254-F259 